\title{
Optimización del uso del paisaje físico-geográfico en las zonas funcionales de la microcuenca Potrero de la Palmita, Nayarit, México
}

\author{
Physical-geographic landscape use optimization in the \\ functional zones of the Potrero de la Palmita \\ micro-basin, Nayarit, Mexico
}

\author{
Alejandro César Valdés Carrera* \\ Juan Alfredo Hernández Guerrero** \\ Julio César Carbajal Monroy***
}

Recibido 26 de septiembre de 2019; aceptado 29 de enero 2020

\section{Resumen}

La planeación del territorio comprendido en las zonas funcionales de una cuenca requiere un enfoque integrador y participativo, desarrollado a partir de una metodología amplia y compleja que considere las cualidades y debilidades del territorio en cuestión, para así tomar la mejor decisión con respecto al uso actual y futuro del paisaje, contemplando sus condiciones biofísicas y socio-productivas. El presente trabajo tiene como objetivo elaborar una propuesta de optimización del uso del paisaje en las zonas funcionales de la microcuenca Potrero de la Palmita, Nayarit, para contribuir a la generación de información cartográfica a escala local, a la planeación y a la gestión de su territorio. Para dar respuesta al objetivo se llevó a cabo un diagnóstico integrado que retomó los métodos de balance hídrico, cambio de uso del suelo, erosión hídrica laminar, susceptibilidad ante peligros naturales y aptitud territorial. Además, se

* Universidad Nacional Autónoma de México (UNAM), México, correo electrónico: alejandrovaldes321@gmail.com. ORCID: https://orcid.org/0000-0002-9480-9331.

** Universidad Autónoma de Querétaro, (UAQ), México, correo electrónico: juan.hernandez@uaq.mx. ORCID: https://orcid.org/0000-0002-4220-7216.

*** Universidad Autónoma del Estado de México (UAEM), México, correo electrónico: jul_geom@hotmail.com. ORCID: https://orcid.org/0000-0003-2303-3756. 
realizó un diagnóstico participativo que consideró el método de grupos focales, talleres y SIG participativos, y finalmente, se propuso el método de optimización del uso del territorio a escala local (1:10 000). La información obtenida fue procesada por medio de una plataforma de Sistemas de Información Geográfica (SIG). El resultado fue la descripción detallada del estado socioambiental de las unidades de paisaje, con base en el cual se propusieron nueve diferentes usos del suelo (Conservación, Conservación-Turístico, Pecuario, Pecuario-Turístico, Agrícola, Agrícola-Turístico, Agropecuario, Agropecuario-Turístico y Asentamiento-Turístico). La investigación representó una metodología viable para abordar la planeación territorial a escala de gran detalle en una microcuenca, permitiendo generar estrategias de gestión, planeación y manejo, a partir de la identificación de su estado, dinámica, procesos, potenciales y problemáticas socioambientales, teniendo como principal herramienta el uso de plataformas de SIG.

Palabras clave: Sistemas de Información Geográfica (SIG), Territorio, Cuenca hidrográfica, Planeación territorial, Paisaje físico-geográfico.

\begin{abstract}
The territorial planning in the basin's functional zones requires an integrative and participatory approach, developed from a complex methodology that considers the qualities and weaknesses of the territory in test, in order to take the best decision regarding the current and future landscape use, contemplating its biophysical and socioproductive conditions. The present work has as objective to elaborate a proposal of landscape use optimization in the functional zones of the Potrero de la Palmita micro-basin, Nayarit, to contribute to the generation of cartographic information at a local scale, to the planning and the management of its territory. In order to answer the main objective, an integrated diagnosis was made, which took up the methods of water balance, land use change, laminar water erosion, susceptibility to natural hazards and territorial aptitude. In addition, a participatory diagnosis was made that considered the method of focal groups, workshops and participatory GIS, and finally, the method of territory use optimization on a local scale (1:10 000) was proposed, the information obtained was processed in a Geographic Information System (GIS) platform. The result was a detailed description of the socio-environmental status of the landscape units, based on which nine different land uses were proposed (Conservation, Conservation-Tourism, Livestock, Livestock-Tourism, Agricultural, Agricultural-Tourism, Agricultural-Livestock, Agricultural-Livestock-Tourism and Settlement-Tourism). The research represented a viable methodology to approach territorial planning at a very detailed scale in a micro-basin, allowing to make management and planning strategies, based on their status, dynamics, processes, potentials and socio-environmental problems, taking into account GIS platforms as main tool.
\end{abstract}


Key words: Geographic Information Systems (GIS), Territory, Hydrographic Basin, Territorial Planning, Physical-Geographic Landscape.

\section{Introducción}

El ordenamiento territorial es una disciplina científica, técnica-administrativa y política, desarrollada en los últimos treinta años del siglo XX para promover el desarrollo "equilibrado y sostenible" de los pueblos, prevenir los conflictos surgidos por la presión sobre los recursos naturales, corregir los efectos de conflictos armados en el planeta y organizar el espacio geográfico (Salinas, 2008).

Representa un conjunto de normas establecidas por un grupo social, destinadas a conseguir la correcta vertebración del territorio, paliando en la medida de lo posible las diferencias socioeconómicas de las poblaciones provocadas por procesos de carácter natural, histórico, social y económico, por lo tanto, la ordenación del territorio necesita de ciertos enfoques y disciplinas que le permitan alcanzar sus objetivos (Noriega, 2008).

El enfoque de cuencas, aporta las herramientas e insumos necesarios a nivel espacial para realizar un ordenamiento del territorio, no solo desde el punto de vista geográfico natural, sino también humano, ya que en él, tienen asiento una complejidad de procesos derivados de las relaciones hombre-naturaleza y hombre-hombre (Arias y Duque, 1992 en García, 2006). Sin embargo, su estudio presenta el reto de delimitar unidades ambientales homogéneas de menor escala, que permitan realizar una caracterización de sus componentes, tomando en cuenta su funcionamiento particular sin perder de vista el lugar que ocupan como componente del sistema llamado cuenca (Cotler, 2007).

Dichas unidades son bien representadas por el enfoque paisajístico físicogeográfico, el cual a través de la clasificación y análisis de las unidades de paisaje, permite la gestión del espacio en un contexto de ordenamiento territorial, donde se puede satisfacer de manera planificada: el desarrollo armónico de la economía, las expectativas sociales y la conservación y manejo del patrimonio natural (Hernández et al., 2012).

El enfoque paisajista ha evolucionado a lo largo del tiempo. Según Bocco et al. (2009), el primer abordaje lo realizó la escuela rusa a finales del siglo XIX, con el "Enfoque de la Geografía Física Compleja o Geografía del Paisaje", generando unidades integrales del espacio geográfico. Seguido de la escuela de la ex Alemania Oriental que para los años 30's propuso el "Enfoque de la Ecología del Paisaje o Geoecología", que refiere a esferas parciales de geocomponentes que conforman geocomplejos (Bocco et al., 2009).

Bocco y colaboradores también refieren a la escuela Australiana la cual propuso el "Levantamiento de Tierras" (40’s), de carácter fisonómico y fisiográfico. En los 
años 70's, la escuela de la antigua Checoslovaquia, propuso el "Enfoque de Planeación Ecológica del Paisaje", delimitando sistemas territoriales socio-naturales. En los años 80’s, el “Levantamiento de Ecología del Paisaje”, consideró las relaciones verticales y horizontales de las unidades de paisaje. El "Levantamiento Geopedológico" de Zinck, clasificó jerárquicamente los suelos, y por ende, los recursos naturales.

Por su parte, la escuela Francesa propuso el "Enfoque Morfopedológico", delimitando unidades resultantes del balance morfogénesis-pedogénesis. La "Regionalización Ecológica" de la Comisión para la Cooperación Ambiental, incorporó criterios ecológicos en las clasificaciones territoriales. Finalmente, a finales de los 90's, la escuela Holandesa por medio del ITC (International Institute for Geo-Information Science and Earth Observation), propuso el "Levantamiento Geomorfológico", delimitando unidades físico-ambientales enfatizando aspectos de geomorfología o uso potencial mediante un enfoque de paisaje (Bocco et al., 2009).

Los anteriores postulados y aproximaciones muestran la evolución del concepto del paisaje, concentrándose las más antiguas en describir las relaciones ambientales del espacio geográfico, evolucionando hasta contemplar unidades socioambientales que requieren de una visión integral de la naturaleza y de las modificaciones del hombre sobre la superficie terrestre, lo cual muestra un marco teórico y conceptual que fundamenta la aplicación del enfoque de paisaje en estudios de planeación y ordenamiento territorial.

De tal forma, el concepto de paisaje puede tomarse como base en los trabajos de planeación territorial en cuencas hidrográficas, ya que, según Vargas (2012), ésta busca racionalizar el uso del espacio por medio de acciones ordenadas, promoviendo la mejor repartición espacial de actividades según los recursos disponibles, ayudándose de herramientas para el manejo de datos cartográficos y el modelado espacial representadas por los SIG, permitiendo a la sociedad confeccionar estrategias y acciones de ordenamiento más acertadas.

En ese sentido, existen ejemplos que fundamentan el trabajo en cuencas a través de un enfoque paisajístico con miras al ordenamiento del territorio. Algunos trabajos destacados se describen a continuación. El análisis del paisaje geosistémico de la cuenca del Río Martín en Aragón, "España”, retomó la metodología de la Geografía física compleja (Franch et al., 2015). Asimismo, Nogué y Sala (2008) realizaron la evaluación del paisaje de Cataluña con el objetivo de ordenar el territorio, desarrollando una metodología que considera primordial el inventario de valores paisajísticos.

En el caribe centroamericano, Hasdenteufel et al. (2008) realizaron el modelo de ordenamiento ambiental de la cuenca del Río Quibú en Cuba mediante un enfoque de gestión ambiental y manejo integrado, siguiendo una metodología geoecológica. En sudamérica, López et al. (2012) efectuaron una zonificación ambiental con miras al ordenamiento y manejo del territorio de la zona costera del departamento del 
Atlántico en Colombia, partiendo de la delimitación de unidades ecológicas del paisaje. Jaque (2010) elaboró el diagnóstico territorial de la cuenca del Río Andalién en Chile, siguiendo el modelo geosistémico o de análisis integrado de sistemas naturales y considerando la identificación de unidades geosistémicas y sus geofacies. Por su parte, Muñoz y Azócar (2014), también en Chile, realizaron la gestión del territorio de la cuenca del Río Baker, basándose en la metodología de análisis y gestión del paisaje. Por último, Bertani (2011), realizó una propuesta de planeación y ordenamiento territorial del departamento de Minas en la Provincia de Neuquén, Argentina, mediante una evaluación geoecológica de los paisajes.

Las aportaciones anteriores demuestran la compatibilidad entre el enfoque paisajista y el enfoque de cuencas, observándose un nicho potencial de acción que permitirá abordar el territorio denominado cuenca por medio de sus zonas funcionales analizándolo a través de las unidades de paisaje físico-geográfico que las constituyen, lo cual no se ha realizado en alguna otra investigación con enfoque de cuencas, paisaje u ordenamiento territorial.

Con ello se puede lograr una mejor gestión y planeación territorial de la cuenca hidrográfica, además de caracterizar de forma detallada sus condiciones ambientales, sociales y económicas, analizar sus problemáticas, diagnosticar su estado actual, incorporar de manera integral temáticas como riesgos naturales, equilibrio hídrico, aptitud, conflictos y potencial turístico entre otras, así como, trabajar a diferentes escalas apoyándose de herramientas cartográficas como los SIG.

La microcuenca Potrero de la Palmita está inmersa dentro de la dinámica actual del estado de Nayarit, el cual busca fortalecer la conectividad y el desarrollo de sus diferentes centros poblacionales urbanos y/o rurales. En este sentido, ha dirigido su política de planeación hacia el desarrollo regional y el crecimiento económico, fomentando la consolidación de sus principales zonas urbanas por medio de desarrollos habitacionales e industriales, el desarrollo de infraestructura (presas), el mejoramiento de vialidades y el apoyo a proyectos ecoturísticos y etnoturísticos en las zonas rurales.

No obstante, dicha política al ser coordinada de forma inadecuada, ha tenido un impacto regional negativo, provocando el incremento en la demanda de recursos naturales como el agua y el suelo, principalmente al centro, este y oeste de la entidad, generando a su vez problemáticas ambientales, sociales y económicas, las cuales se acentúan a escala local repercutiendo directamente en la microcuenca.

Esto ha detonado problemáticas ambientales (degradación y contaminación), sociales (migración) y económicas (pobreza-marginación) dentro de su territorio. A pesar de esto, la microcuenca no cuenta con un estudio que aborde su estado actual y considere sus potenciales y problemáticas mediante un enfoque de planeación territorial local, por lo que representa una zona vulnerable al efecto de políticas regionales y a procesos como la erosión, la remoción en masa y los cambios de uso del suelo. 
Por lo comentado hasta el momento, la presente investigación tiene como objetivo principal proponer una estrategia de optimización del uso del paisaje físicogeográfico en las zonas funcionales de la microcuenca Potrero de la Palmita basado en la construcción de un Sistema de Información Geográfica territorial, el cual contribuirá de manera sustancial en la planeación, gestión y manejo de su territorio a escala local.

\section{Área de estudio}

La microcuenca Potrero de la Palmita, se ubica al oeste de la República Mexicana, dentro del municipio del Nayar, localizado al este del estado de Nayarit. Pertenece a la región hidrológica número 12 Lerma-Chapala-Santiago, a su vez, a la cuenca del Río Santiago-Aguamilpa y a la subcuenca del Río Grande de Santiago, cubriendo una superficie de 473 hectáreas.

En la microcuenca Potrero de la Palmita, habita el grupo étnico wixárika o huichol (etnia de la región centro-oeste de México), el cual es reconocido administrativa-

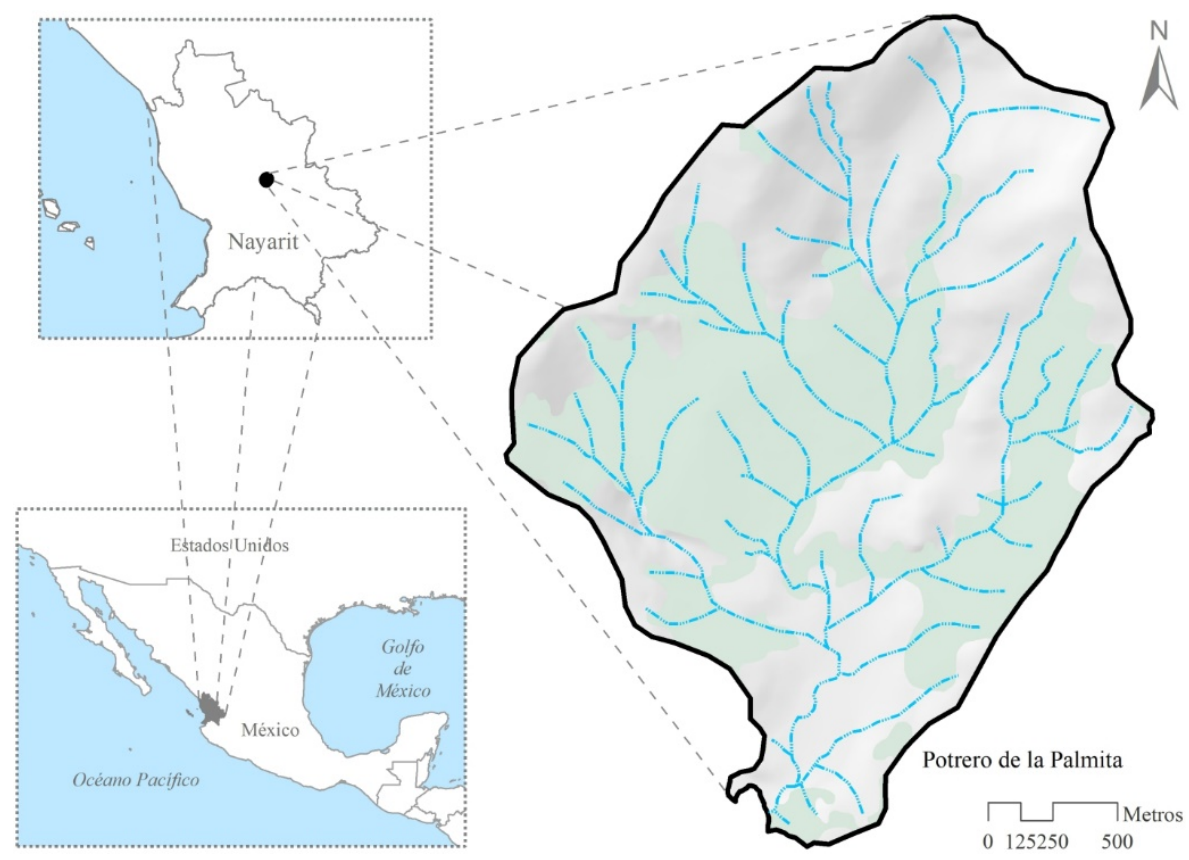

Figura 1. Localización de la microcuenca Potrero de la Palmita. 
mente por el gobierno del municipio del Nayar como la localidad Potrero de la Palmita, quedando asentada cerca de la desembocadura de la microcuenca, zona donde vierte sus aguas al Río Huaynamota. Se sitúa entre los 752 y $210 \mathrm{msnm}$, resaltando elevaciones como el Cerro Cuate (540 msnm) y Peñasquillo (740 msnm), (Figura 1).

Debido a sus características topográficas, se desarrollan diversos procesos geomorfológicos que derivan de forma indistinta en la dinámica biofísica y socioeconómica según la zona funcional, sea a través de múltiples condiciones de deterioro y/o calidad de la estructura vertical (rocas, suelos, condiciones hídricas y vegetación), y horizontal del paisaje físico-geográfico de la microcuenca.

De acuerdo con Valdés y Hernández (2018), la zona funcional media constituye el área de mayor superficie dentro de la microcuenca con 235.98 ha, disgregándose en 18 unidades de paisaje que están representadas por complejos cumbrales y complejos de laderas y barrancos, en ella se observan sitios que van de muy planos a muy inclinados, cuya pendiente oscila entre $1^{\circ} \mathrm{y}>30^{\circ}$, influenciados por un clima cálido subhúmedo que favorece procesos de erosión y transporte. Lo anterior se acompaña de una estructura litológica representada por toba riolítica e ignimbrita, suelos leptosoles, regosoles y feozems, así también por usos de suelo como selva baja caducifolia (77.87\%), zonas agrícolas (13.20\%), pastizales (4.26\%) y áreas para la construcción de viviendas $(4.67 \%)$.

Por otra parte, la zona funcional alta representa el área con la segunda mayor superficie dentro de la microcuenca (190.04 ha), dividiéndose en 17 unidades de paisaje que se constituyen por complejos cumbrales y complejos de laderas y barrancos con pendientes que van de poco inclinadas $\left(5^{\circ}\right)$ a muy inclinadas $\left(>30^{\circ}\right)$, es la zona con mayor presencia de procesos erosivos que actúan sobre riolita, toba riolítica e ignimbrita. Dicho proceso da pie a la existencia de suelos poco profundos como el leptosol y el regosol que, bajo el efecto del mismo clima, permiten el establecimiento de ecosistemas como el bosque de latifoliadas-encino (1.97\%) y selva baja caducifolia $(72.03 \%)$, esos suelos al ser sometidos a usos agropecuarios, dan paso a zonas cubiertas por pastos $(25.58 \%)$ y a reducidos manchones agrícolas $(0.41 \%)$.

Finalmente, la zona baja presenta la menor superficie (47.13 ha) y se conformó por cuatro unidades de paisaje. Respecto a sus geoformas (complejos de laderas y barrancos y complejos de superficies y cauces), la zona está constituida por sitios predominantemente planos $\left(1^{\circ}\right.$ a $\left.3^{\circ}\right)$, receptores de materiales y sedimentos provenientes de la zona alta y media, beneficiando así, el desarrollo de suelos profundos como el feozem y en pequeñas superficies regosoles.

Su basamento y clima son equiparables a las zonas funcionales alta y media, sin embargo, al ser la zona con mayor accesibilidad se concentran en ella los asentamientos humanos $(61.79 \%)$ y las actividades productivas agropecuarias $(31.45 \%)$, reduciendo la cobertura del ecosistema natural característico de la microcuenca, sea la selva baja caducifolia $(6.77 \%)$. 


\section{Métodos y herramientas}

\section{Diagnóstico integrado de unidades de paisaje}

El proceso se desarrolló a partir de cinco fases: 1) balance hídrico; 2) cambio de uso del suelo (1974-2016); 3) erosión hídrica laminar; 4) susceptibilidad ante peligros naturales; y 5) aptitud territorial. Cabe señalar que el balance hídrico toma de base los aportes de la Comisión Nacional del Agua enunciados en la NOM-011CONAGUA-2015 publicado en el Diario Oficial de la Federación (2015).

El balance hídrico superficial consideró las entradas de agua representadas por el escurrimiento natural por cuenca propia $(C p)$, las importaciones de agua de cuencas vecinas $(\mathrm{Im})$ y los retornos de agua $(R e)$. También se utilizaron las salidas de agua a través de los usos del agua $(U)$ y el escurrimiento a la salida de la cuenca $(A b)$. El balance hídrico se obtuvo con la siguiente expresión:

$$
\mathrm{BH}=\text { Entradas }- \text { Salidas }=\mathrm{BH}=(\mathrm{Cp}+\mathrm{Im}+\mathrm{Re})-(\mathrm{Ab}+\mathrm{U}) .
$$

El cambio de uso del suelo contempló el método de Palacio et al. (2004), mostrando la dinámica de los ecosistemas dentro de un periodo de tiempo determinado, permitiendo identificar el grado de perturbación y/o recuperación de los mismos, clasificando el resultado en cinco categorías:

1. Conservada: cobertura natural que no ha sufrido cambio en el periodo;

2. Revegetada: zona actualmente ocupada por bosque, selva, matorral, vegetación hidrófila o pastizal natural, donde anteriormente se desarrollaba alguna actividad agropecuaria (cultivos o pastizales);

3. Deforestada: corresponde con la superficie arbórea (bosques y selvas), que ha cambiado a zonas de cultivos, pastizales inducidos o a uso urbano;

4. Por actividad productiva: considera la superficie donde anteriormente presentaba cobertura de origen antrópico como: pastizales inducidos, cultivados, algún tipo de uso de suelo urbano, cuerpo de agua, que actualmente ha cambiado de actividad productiva; $y$

5. Sin cambio: áreas con cultivos, pastizales inducidos y cultivados, y otros usos, que actualmente se mantienen con el mismo tipo de uso.

La erosión hídrica laminar retomó la Ecuación Universal de Pérdida de Suelo (USLE) elaborada por Wischmeier y Smith (1978, citada en Colín et al., 2013), la cual contabiliza la tasa la pérdida de suelo (A) en toneladas por hectárea al año (ton/ha/año) y considera el factor R (erosividad de la lluvia), (Mj/ha mm/hr), el factor $\mathrm{K}$ (erodabilidad del suelo), el factor LS (longitud y el grado de pendiente), y el factor $\mathrm{C}$ (protección de la vegetación). La expresión matemática que se utilizó fue $\mathrm{A}=\mathrm{R} * \mathrm{~K} * \mathrm{LS} * \mathrm{C}$. Además, con base en Martínez (2005), se clasificaron los resultados en cuatro grados de erosión: 1) Nula o ligera $(0-10)$; 2) Moderada (10-50); 3) Alta (50-200); y 4) Muy alta (>200). 
En cuanto a la susceptibilidad ante peligros naturales, se consideró el método de Reyes y Campos (2014), quienes reconocen las zonas susceptibles ante procesos geológicos e hidrometeorológicos, por lo cual, se generó información para remoción en masa, erosión hídrica e inundaciones, partiendo de la definición basada en bibliografía de peligro, seguida de la identificación y de la ponderación de los componentes ambientales que los condicionan, clasificados según su grado de susceptibilidad en las categorías de Muy alta, Alta, Media, Baja, Muy baja y Nula.

Finalmente, la aptitud territorial se llevó a cabo con el método de Bocco et al. (2010), con el cual se identificaron las zonas con mayor o menor potencial natural ante el desarrollo de alguna cobertura, servicio o actividad productiva, evaluando la aptitud con dos fines: 1) ambientales: consideró la aptitud forestal natural y para la prestación de servicios ambientales; y 2) socio-productivos: contemplando la aptitud para el cultivo de nopal, para la agricultura de temporal, para ganadería extensiva, para el turismo y para el establecimiento de asentamientos humanos. El desarrollo del método inició con la descripción del tipo de aptitud (basada en la bibliografía), después se llevó a cabo la identificación y ponderación de los componentes ambientales que la condicionan, y en último lugar se clasificó conforme a su grado de aptitud en las categorías de muy alta, alta, media, baja, muy baja y nula.

\section{Diagnóstico participativo de las unidades de paisaje}

Esta fase se desarrolló en tres etapas: 1) presentación y conformación del grupo focal; 2) salidas de campo; y 3) Taller y SIG participativo. Para ello, se retomó el método de Geilfus (2009) y el de Fernández et al. (2009) adaptándolos para trabajar a nivel microcuenca.

La primera etapa inició con la presentación del interventor y la selección del grupo focal conformado por un mínimo de cuatro y un máximo de 12 integrantes de la comunidad con representatividad de género y edad, quienes tuvieron especial interés en trabajar y plasmar sus ideas con respecto a las problemáticas y potenciales de su territorio. En la segunda etapa se realizaron recorridos de campo con los integrantes del grupo focal, llevando a cabo dos salidas, una en temporada de lluvias y otra en temporada de sequía, ambas con el objetivo de identificar problemáticas y potenciales.

En la tercera etapa se llevó a cabo un taller participativo donde se registró y priorizó la percepción del grupo focal respecto a las problemáticas y potenciales socioambientales, apoyados de un formato de registro (cuestionario) donde se puntualizaron las posibles acciones de aprovechamiento y/o corrección de las mismas. A partir de esa actividad, se desarrolló el SIG participativo que consistió en la representación de las problemáticas y potenciales en un mapa de su territorio, mediante dibujos y trazos y después se incluyó en el SIG. 
Finalmente, a través de ambos productos se reforzó el trabajo realizado y resumiendo ante los asistentes los resultados obtenidos, se llegó a un acuerdo en común con respecto a las temáticas abordadas.

Los diagnósticos (integrado y participativo) de las unidades de paisaje fueron abordados por medio de técnicas de análisis cartográfico, multicriterio, álgebra de mapas, trabajo de campo, grupos focales y SIG participativo, considerando los archivos en formato vectorial y raster referentes a los factores de cada tema y a las percepciones del grupo focal con respecto a la temática del taller como variables de análisis, las cuales fueron procesadas mediante formatos de registro, cartografía impresa, el manejador de bases de datos Excel y la plataforma de SIG, ArcGIS (v.10.3).

\section{Propuesta de optimización del uso del territorio}

La propuesta se llevó a cabo mediante el análisis detallado de las condiciones presentes en las unidades de paisaje físico-geográfico que conforman las zonas funcionales de la microcuenca, para lo cual, se construyó una base de datos que priorizó los resultados del diagnóstico (integrado y participativo) por unidad de paisaje.

Se consideró como primer filtro a la unidad de paisaje representada por complejos cumbrales, complejos de laderas y barrancos y/o complejos de superficies y cauces, con pendiente entre $1^{\circ} \mathrm{y}>30^{\circ}$, con basamento rocoso de riolita, toba riolítica o ignimbrita, clima cálido subhúmedo, con suelos leptosol, regosol y/o feozem; y cobertura de selva baja caducifolia, bosque natural de latifoliadas-encino, pastizal inducido, agricultura de temporal y/o asentamientos humanos.

Respecto al balance hídrico, se definieron valores mayores a $10000 \mathrm{~m}^{3} / \mathrm{año} \mathrm{de}$ volumen de escurrimiento superficial $(>)$ y valores menores a $10000 \mathrm{~m}^{3} /$ año $(<)$. Para el cambio de uso del suelo valores mayores a $0.1 \%$ de la categoría de "deforestación", de no presentarla, la categoría con predominio en más del $50 \%$ de su superficie o con los valores más cercanos a 50\%; para la erosión hídrica laminar (USLE), a la categoría con predominio en más del $40 \%$ de su superficie.

Para la susceptibilidad ante inundaciones, se contempló sólo la categoría predominante en más del 50\% de su superficie. Para la susceptibilidad por remoción en masa y por erosión, al igual que para los resultados de aptitud (forestal natural, prestación de servicios ambientales, cultivo de nopal, agrícola de temporal, ganadera extensiva, turística y para el establecimiento de asentamientos humanos), se consideró a la categoría que reportó los valores más cercanos al 50\%, o bien, con predominio en más del 50\% de su superficie.

En cuanto al potencial natural, paisajístico, cultural-educativo, turístico y económico, así como para las problemáticas de falta de agua y pérdida de cultivos, erosión hídrica y remoción en masa, degradación y contaminación, migración y desempleo, pérdida de cultura e identidad comunitaria y dotación de servicios, se contempló la detección realizada por la comunidad. 
Posteriormente, se identificó el uso del suelo principal, secundario y terciario presente en cada unidad de paisaje, el cual fue comparado con los componentes de la estructura vertical y los resultados del diagnóstico integrado y participativo priorizados, determinando así su compatibilidad (alta, media o baja). Acto seguido se propusieron los usos del suelo óptimos a desarrollar para el mejor funcionamiento y reducción de problemáticas presentes en cada unidad de paisaje (Tabla 1).

Tabla 1

Usos propuestos para la optimización del territorio

\begin{tabular}{ll}
\hline \multicolumn{1}{c}{ Uso } & \multicolumn{1}{c}{ Descripción } \\
\hline Conservación & $\begin{array}{l}\text { Permanencia de la selva baja caducifolia y bosque natural de } \\
\text { latifoliadas-encino, en las unidades de paisaje, fomentando con } \\
\text { ello la protección del suelo, la mejora del hábitat de flora y } \\
\text { fauna, el aprovechamiento del ciclo del agua, procurando la } \\
\text { recarga y captación superficial y la producción de microclimas }\end{array}$ \\
Desarrollo de actividades ecoturísticas y etnoturísticas en las \\
unidades de paisaje que concentran los ecosistemas naturales de \\
Turístico & $\begin{array}{l}\text { bosque y selva } \\
\text { Se llevan a cabo actividades de pastoreo del ganado (vacas, } \\
\text { Pecuario }\end{array}$ \\
borregos, cabras) en unidades de paisaje con vegetación de \\
pastizal \\
Implementación de actividades ecoturísticas y etnoturísticas en \\
las unidades de paisaje que concentran las actividades de \\
pastoreo \\
Establecimiento de cultivos de maíz de temporal (milpa \\
tradicional) y/o cultivos de nopal en las unidades de paisaje \\
donde sea posible obtener mejores rendimientos para \\
autoconsumo \\
Agrícola
\end{tabular}

Fuente: elaboración propia. 


\section{Resultados}

\section{Diagnóstico integrado y participativo de las unidades de paisaje físico-geográfico por zona funcional}

\section{Zona funcional alta}

Esta zona ocupa el segundo lugar respecto a la cantidad de agua de lluvia que recibe (39.64 \%), la cual se convierte en escurrimientos superficiales que, al vincularse con las actividades productivas, ocasiona importantes problemáticas de erosión hídrica laminar, pues al año genera la pérdida de más de 200 toneladas de suelo por hectárea, al tiempo que los resultados del modelo USLE señalaron que ese fenómeno predomina en más del 90\% de la superficie de la zona (Figura 2).

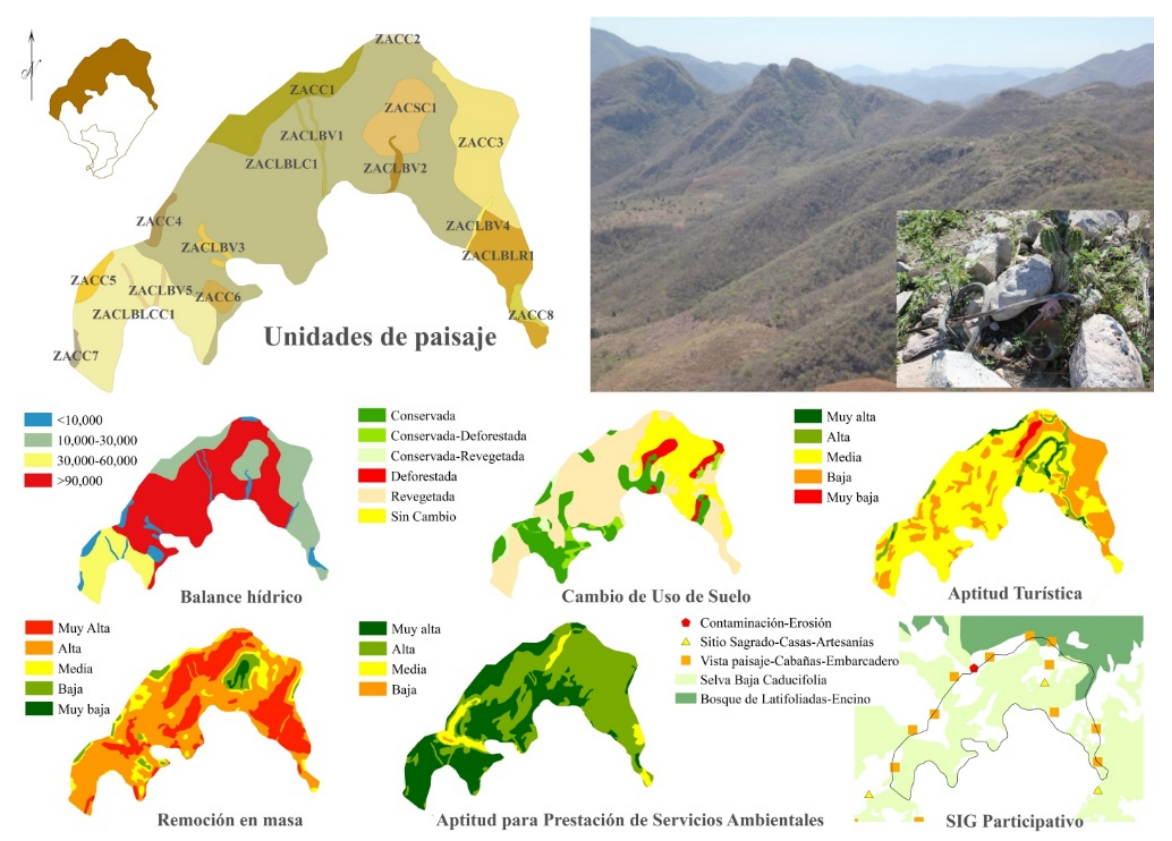

Figura 2. Diagnóstico integrado y participativo de la zona funcional alta.

También, representa la zona con mayor susceptibilidad ante peligros geológicos, ya que en más del $80 \%$ de su área se identificaron valores altos de remoción en masa y erosión. Por otra parte, es la zona con menor susceptibilidad ante peligros hidrometeorológicos, puesto que más del 95\% presenta valores nulos ante inundaciones. Entre los años de 1974 y 2016 el cambio de uso del suelo en esta zona se manifestó, de forma general, en la disminución de zonas de pastizal, beneficiando la recuperación 
de las coberturas naturales representadas por la selva baja caducifolia y el bosque natural de latifoliadas-encino.

A pesar de las problemáticas señaladas, la zona cuenta con potenciales asociados a la selva baja caducifolia (en más del 93\%), sea la prestación de servicios ecosistémicos, conservación de suelos, recarga hídrica, protección y conservación de la biodiversidad. Por otro lado, es la zona con menor aptitud para el desarrollo de asentamientos humanos y actividades económicas. Al respecto, la evaluación señala que el establecimiento de asentamientos humanos resultó con valores nulos en más del $60 \%$ y bajos en más del $24 \%$ de su superficie.

Asimismo, la agricultura de temporal presenta valores nulos en más del $50 \%$ y valores bajos en más del 30\%, mientras que el desarrollo de ganadería extensiva reporta valores bajos en más del $85 \%$ de su superficie. Lo anterior significa que dichas actividades deben ser limitadas o evitadas para conservar el funcionamiento de la zona. A pesar de lo señalado, se identificó aptitud para el desarrollo de actividades turísticas con valores medios en más del $60 \%$ de su área (Tabla 2).

Tabla 2

Diagnóstico integrado para la zona funcional alta

\begin{tabular}{|c|c|c|c|c|c|c|c|c|c|c|c|c|c|}
\hline \multirow{3}{*}{$\begin{array}{c}\text { Clave de la } \\
\text { unidad de } \\
\text { paisaje }\end{array}$} & \multicolumn{13}{|c|}{ Diagnóstico integrado } \\
\hline & \multirow[b]{2}{*}{$\mathbb{\pi}$} & \multirow[b]{2}{*}{$\tilde{U}$} & \multirow{2}{*}{ 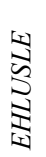 } & \multicolumn{3}{|c|}{ Susceptibilidad } & \multicolumn{7}{|c|}{ Aptitud } \\
\hline & & & & ऐ & 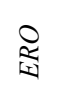 & $\gtreqless$ & 廷 & है & క & ษ & 선 & $\pi$ & $\underset{\mathbb{N}}{\mathbb{1}}$ \\
\hline ZACC1 & $>$ & $\mathrm{R}$ & $\mathrm{A}$ & $\mathrm{A}$ & $\mathrm{M}$ & $\mathrm{N}$ & $\mathrm{A}$ & $\mathrm{A}^{\prime}$ & $\mathrm{M}$ & $\mathrm{N}$ & $\mathrm{B}^{\prime}$ & $\mathrm{M}$ & $\mathrm{N}$ \\
\hline ZACC2 & $<$ & $\mathrm{R}$ & $A^{\prime}$ & A & $\mathrm{M}$ & $\mathrm{N}$ & $\mathrm{A}^{\prime}$ & $A^{\prime}$ & $\mathrm{M}$ & $\mathrm{N}$ & $\mathrm{B}$ & $\mathrm{M}$ & $\mathrm{N}$ \\
\hline ZACC3 & $>$ & D & $A^{\prime}$ & A & A & $\mathrm{N}$ & A & A & A & $\mathrm{N}$ & B & B & $\mathrm{N}$ \\
\hline ZACC4 & $<$ & $\mathrm{R}$ & A & M & M & $\mathrm{N}$ & A & M & A & $\mathrm{N}$ & B & B & B' \\
\hline ZACC5 & $<$ & $\mathrm{C}$ & A & A & M & $\mathrm{N}$ & A & $A^{\prime}$ & $\mathrm{M}$ & $\mathrm{N}$ & B & $\mathrm{M}$ & $\mathrm{N}$ \\
\hline ZACC6 & $<$ & $\mathrm{C}$ & A & $\mathrm{M}$ & $\mathrm{M}$ & $\mathrm{N}$ & $\mathrm{A}^{\prime}$ & $A^{\prime}$ & $\mathrm{M}$ & $\mathrm{B}^{\prime}$ & B & $\mathrm{M}$ & $\mathrm{N}$ \\
\hline ZACC7 & $<$ & $\mathrm{R}$ & $A^{\prime}$ & A & $\mathrm{M}$ & $\mathrm{N}$ & $A^{\prime}$ & $A^{\prime}$ & $\mathrm{M}$ & $\mathrm{B}^{\prime}$ & B & $\mathrm{M}$ & B' \\
\hline ZACC8 & $<$ & $\mathrm{R}$ & $A^{\prime}$ & A & A & $\mathrm{N}$ & $\mathrm{A}^{\prime}$ & A & A & B & B & B & $\mathrm{N}$ \\
\hline ZACLBLC1 & $>$ & $\mathrm{D}$ & $A^{\prime}$ & A & $\mathrm{M}$ & $\mathrm{N}$ & $\mathrm{A}^{\prime}$ & A & A & $\mathrm{N}$ & B & $\mathrm{M}$ & $\mathrm{N}$ \\
\hline ZACLBLCC1 & $>$ & $\mathrm{C}$ & $A^{\prime}$ & A & M & $\mathrm{N}$ & A & $A^{\prime}$ & A & $\mathrm{N}$ & B & M & $\mathrm{N}$ \\
\hline ZACLBLR1 & $>$ & $\mathrm{R}$ & $A^{\prime}$ & $A^{\prime}$ & A & $\mathrm{N}$ & A & A & A & $\mathrm{N}$ & B & B & $\mathrm{B}^{\prime}$ \\
\hline ZACLBV1 & $<$ & $\mathrm{R}$ & $A^{\prime}$ & A & $\mathrm{M}$ & $\mathrm{N}$ & A & A & M & $\mathrm{N}$ & B & M & $\mathrm{N}$ \\
\hline ZACLBV2 & $<$ & $\mathrm{D}$ & $A^{\prime}$ & A & A & $\mathrm{N}$ & A & $A^{\prime}$ & B & $\mathrm{N}$ & A & M & $\mathrm{N}$ \\
\hline ZACLBV3 & $<$ & $\mathrm{C}$ & $A^{\prime}$ & A & M & $\mathrm{N}$ & A & $A^{\prime}$ & M & $\mathrm{N}$ & A & M & $\mathrm{N}$ \\
\hline ZACLBV4 & $<$ & $\mathrm{C}$ & $A^{\prime}$ & A & M & $\mathrm{N}$ & A & A & M & $\mathrm{N}$ & B & M & $\mathrm{N}$ \\
\hline ZACLBV5 & $<$ & $\mathrm{C}$ & $\mathrm{A}^{\prime}$ & A & M & $\mathrm{N}$ & A & A & B & $\mathrm{N}$ & B & M & $\mathrm{N}$ \\
\hline ZACSC1 & $>$ & $\mathrm{D}$ & $\mathrm{A}$ & $\mathrm{B}$ & $\mathrm{B}$ & $\mathrm{B}$ & $\mathrm{A}^{\prime}$ & A & $\mathrm{A}^{\prime}$ & $\mathrm{A}^{\prime}$ & A & $\mathrm{M}$ & A \\
\hline \multicolumn{14}{|c|}{$\begin{array}{l}\text { BH: balance hídrico, >: mayor a } 10000 \mathrm{~m}^{3} / \text { año, }<: \text { menor a } 10000 \mathrm{~m}^{3} / \text { año CUS: cambio de uso del } \\
\text { suelo, R: revegetada, D: deforestada, C: conservada; EHLUSLE: erosión hídrica laminar USLE, RM: } \\
\text { remoción en masa, ERO: erosión, INU: inundaciones, FN: forestal natural, PSA: prestación de servi- } \\
\text { cios ambientales, CN: cultivo de nopal, AT: agrícola de temporal, GE: ganadera extensiva, T: turística, } \\
\text { EAH: establecimiento de asentamientos humanos; A': muy alta, A:alta, M: media, B: baja, B': muy } \\
\text { baja, N: nula. }\end{array}$} \\
\hline
\end{tabular}

Fuente: elaboración con base en los resultados del SIG y de campo. 
Los resultados del diagnóstico participativo indican que la zona funcional alta es percibida por la comunidad por su potencial natural (flora y fauna), paisajístico (bellas vistas del territorio) y cultural-educativo (alberga sitios tradicionales y religiosos para la etnia), todo ello le imprime un alto valor turístico y económico que permitiría su aprovechamiento como insumo de materias primas, panorámicas y rituales para ser otorgados a los visitantes.

En otro contexto, se señalaron problemáticas como falta de agua, pérdida de cultivos derivada de la escasez de lluvia, erosión, remoción en masa, degradación de suelos y vegetación, y contaminación. Esas problemáticas serían mitigadas con la construcción de pequeños cuerpos de agua, la disminución de la quema y el desmonte al abrir nuevas zonas de cultivo y pastoreo, al evitar la disposición y quema inadecuada de residuos sólidos, y al evitar la aplicación de herbicidas en las milpas (Tabla 3).

Tabla 3

Diagnóstico participativo para la zona funcional alta

\begin{tabular}{|c|c|c|c|c|c|c|c|c|c|c|c|c|}
\hline \multirow{3}{*}{$\begin{array}{c}\text { Zona } \\
\text { Funcional }\end{array}$} & \multirow{3}{*}{ Clave UP } & \multicolumn{11}{|c|}{ Diagnóstico participativo } \\
\hline & & \multicolumn{5}{|c|}{ Potencial } & \multicolumn{6}{|c|}{ Problemática } \\
\hline & & $N$ & $C E$ & $P$ & $T U$ & $E$ & $A P C$ & $E R$ & $D C$ & $M D$ & $C I$ & $S$ \\
\hline \multirow{17}{*}{ Alta } & ZACC1 & $\mathrm{O}$ & & $\mathrm{O}$ & & & & $\mathrm{O}$ & & & & \\
\hline & ZACC2 & $\mathrm{O}$ & & $\mathrm{O}$ & & & & & & & & \\
\hline & ZACC3 & $\mathrm{O}$ & & $\mathrm{O}$ & & & & & & & & \\
\hline & ZACC4 & $\mathrm{O}$ & & $\mathrm{O}$ & $\mathrm{O}$ & $\mathrm{O}$ & & & & & & \\
\hline & ZACC5 & $\mathrm{O}$ & & $\mathrm{O}$ & & & & & & & & \\
\hline & ZACC6 & $\mathrm{O}$ & & $\mathrm{O}$ & & & & & & & & \\
\hline & ZACC7 & $\mathrm{O}$ & & $\mathrm{O}$ & & & & & & & & \\
\hline & ZACC8 & & & & & & & & & & & \\
\hline & ZACLBLC1 & $\mathrm{O}$ & & $\mathrm{O}$ & $\mathrm{O}$ & $\mathrm{O}$ & $\mathrm{O}$ & $\mathrm{O}$ & $\mathrm{O}$ & & & \\
\hline & ZACLBLCC1 & $\mathrm{O}$ & $\mathrm{O}$ & $\mathrm{O}$ & $\mathrm{O}$ & $\mathrm{O}$ & $\mathrm{O}$ & & $\mathrm{O}$ & & & \\
\hline & ZACLBLR1 & $\mathrm{O}$ & & $\mathrm{O}$ & $\mathrm{O}$ & $\mathrm{O}$ & & & & & & \\
\hline & ZACLBV1 & $\mathrm{O}$ & & $\mathrm{O}$ & & & & & & & & \\
\hline & ZACLBV2 & $\mathrm{O}$ & & $\mathrm{O}$ & & & $\mathrm{O}$ & & $\mathrm{O}$ & & & \\
\hline & ZACLBV3 & $\mathrm{O}$ & & $\mathrm{O}$ & & & & & & & & \\
\hline & ZACLBV4 & $\mathrm{O}$ & & $\mathrm{O}$ & & & & & & & & \\
\hline & ZACLBV5 & $\mathrm{O}$ & & $\mathrm{O}$ & & & & & & & & \\
\hline & ZACSC1 & $\mathrm{O}$ & $\mathrm{O}$ & $\mathrm{O}$ & $\mathrm{O}$ & $\mathrm{O}$ & & & & & & \\
\hline
\end{tabular}

N: natural, CE: cultural-educativo, P: paisajístico, TU: turístico, E: económico; APC: falta de agua y pérdida de cultivos, ER: erosión hídrica y remoción en masa, DC: degradación y contaminación, MD: migración y desempleo, CI: pérdida de cultura e identidad comunitaria, S: dotación de servicios; O: detectado.

Fuente: $\quad$ elaboración con base en taller y SIG participativo. 


\section{Zona funcional media}

La dinámica de cambio de uso del suelo comprendida entre los años de 1974 y 2016 indica una coexistencia entre la selva baja caducifolia, pastizales inducidos, zonas agrícolas de temporal y asentamientos humanos, predominando las coberturas naturales sobre las antrópicas (Tabla 4).

Tabla 4

Diagnóstico integrado para la zona funcional media

\begin{tabular}{|c|c|c|c|c|c|c|c|c|c|c|c|c|c|}
\hline \multirow{3}{*}{$\begin{array}{c}\text { Clave de la } \\
\text { unidad de } \\
\text { paisaje }\end{array}$} & \multicolumn{13}{|c|}{ Diagnóstico integrado } \\
\hline & \multirow[b]{2}{*}{$\underset{\nwarrow}{\nwarrow}$} & \multirow[b]{2}{*}{$\underset{U}{0}$} & \multirow{2}{*}{ 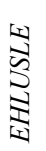 } & \multicolumn{3}{|c|}{ Susceptibilidad } & \multicolumn{7}{|c|}{ Aptitud } \\
\hline & & & & $\gtreqless$ & $\underset{\substack{1 \\
\text { in }}}{0}$ & $\gtreqless$ & 廷 & స్ & ¿ & ซ & 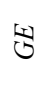 & H & $\underset{\mathbb{4}}{\mathbb{7}}$ \\
\hline ZMCC1 & $<$ & $\mathrm{D}$ & A & A & $\mathrm{M}$ & $\mathrm{N}$ & A & $A^{\prime}$ & A & $\mathrm{N}$ & B & $\mathrm{M}$ & $\mathrm{N}$ \\
\hline ZMCC2 & $<$ & $\mathrm{P}^{\prime}$ & A & $\mathrm{B}$ & $\mathrm{B}$ & $\mathrm{N}$ & M & A & M & $\mathrm{N}$ & $\mathrm{B}^{\prime}$ & A & $\mathrm{B}^{\prime}$ \\
\hline ZMCC3 & $<$ & $\mathrm{R}$ & A & M & M & $\mathrm{N}$ & $A^{\prime}$ & $A^{\prime}$ & A & $\mathrm{B}$ & B & B & $\mathrm{B}^{\prime}$ \\
\hline $\mathrm{ZMCC} 4$ & $<$ & $\mathrm{R}$ & A & B & M & $\mathrm{N}$ & A & A & A & $\mathrm{B}$ & B & M & $\mathrm{N}$ \\
\hline ZMCC5 & $<$ & $\mathrm{R}$ & A & $\mathrm{B}$ & M & $\mathrm{N}$ & A & $A^{\prime}$ & A & $\mathrm{B}$ & B & M & $\mathrm{N}$ \\
\hline ZMCC6 & $<$ & $\mathrm{R}$ & $M^{\prime}$ & B & B & $\mathrm{N}$ & $A^{\prime}$ & $A^{\prime}$ & M & $\mathrm{B}^{\prime}$ & B & A & $\mathrm{N}$ \\
\hline ZMCLBLC1 & $>$ & $\mathrm{D}$ & $A^{\prime}$ & A & M & $\mathrm{N}$ & $A^{\prime}$ & $A^{\prime}$ & A & M & $\mathrm{B}$ & M & M \\
\hline ZMCLBLC2 & $>$ & $\mathrm{C}$ & $A^{\prime}$ & $A^{\prime}$ & A & $\mathrm{N}$ & A & A & A & $\mathrm{N}$ & $\mathrm{B}^{\prime}$ & M & $\mathrm{N}$ \\
\hline ZMCLBLC3 & $>$ & $\mathrm{D}$ & $A^{\prime}$ & $A^{\prime}$ & A & $\mathrm{N}$ & A & A & A & $\mathrm{N}$ & $\mathrm{B}^{\prime}$ & M & $\mathrm{N}$ \\
\hline ZMCLBLC4 & $>$ & $\mathrm{D}$ & $A^{\prime}$ & A & M & $\mathrm{N}$ & A & A & A & B & B & M & $\mathrm{N}$ \\
\hline ZMCLBLC5 & $<$ & $\mathrm{D}$ & $A^{\prime}$ & A & M & $\mathrm{N}$ & $A^{\prime}$ & $A^{\prime}$ & M & $\mathrm{N}$ & B & M & $\mathrm{B}^{\prime}$ \\
\hline ZMCLBLC6 & $>$ & $\mathrm{D}$ & $A^{\prime}$ & A & M & $\mathrm{N}$ & $A^{\prime}$ & $A^{\prime}$ & A & M & B & M & $B^{\prime}$ \\
\hline ZMCLBLCC1 & $>$ & $\mathrm{C}$ & $A^{\prime}$ & A & M & $\mathrm{N}$ & A & $A^{\prime}$ & A & B & B & M & $\mathrm{N}$ \\
\hline ZMCLBLCC2 & $>$ & $\mathrm{D}$ & $A^{\prime}$ & A & M & $\mathrm{N}$ & A & $A^{\prime}$ & A & B & B & M & $\mathrm{N}$ \\
\hline ZMCLBLR1 & $>$ & $\mathrm{R}$ & $A^{\prime}$ & A & M & $\mathrm{N}$ & $A^{\prime}$ & A & A & $\mathrm{B}^{\prime}$ & $\mathrm{B}$ & M & $\mathrm{N}$ \\
\hline ZMCLBV1 & $>$ & $\mathrm{D}$ & $A^{\prime}$ & A & M & $\mathrm{N}$ & A & $A^{\prime}$ & M & $\mathrm{N}$ & A & M & $\mathrm{N}$ \\
\hline ZMCLBV2 & $<$ & $\mathrm{C}$ & $A^{\prime}$ & A & B & $\mathrm{N}$ & A & $A^{\prime}$ & M & $\mathrm{N}$ & A & M & $\mathrm{N}$ \\
\hline ZMCLBV3 & $<$ & $\mathrm{D}$ & $A^{\prime}$ & A & M & $\mathrm{N}$ & A & $A^{\prime}$ & B & $\mathrm{N}$ & A & $\mathrm{M}$ & $\mathrm{N}$ \\
\hline
\end{tabular}

BH: balance hídrico, >: mayor a $10000 \mathrm{~m}^{3} / \mathrm{año,}<$ : menor a $10000 \mathrm{~m}^{3} /$ año; CUS: cambio de uso del suelo, R: revegetada, D: deforestada, C: conservada, P': por actividad productiva; EHLUSLE: erosión hídrica laminar USLE, RM: remoción en masa, ERO: erosión, INU: inundaciones, FN: forestal natural, PSA: prestación de servicios ambientales, CN: cultivo de nopal, AT: agrícola de temporal, GE: ganadera extensiva, T: turística, EAH: establecimiento de asentamientos humanos; A': muy alta, A:alta, M': moderada, M: media, B: baja, B': muy baja, N: nula.

Fuente: elaboración con base en los resultados del SIG y de campo.

La mayor cantidad de agua proveniente de la precipitación pluvial se convierte en escurrimiento (49.94\%), ocupa la segunda posición en cuanto a la problemática de pérdida de suelo según la USLE, ya que presentó en más del 70\% de su superficie 
una tasa mayor a 200 ton/ha/año, condición que se refleja en los valores altos de susceptibilidad ante remoción en masa (70\%) y erosión (30\%), (Figura 3).

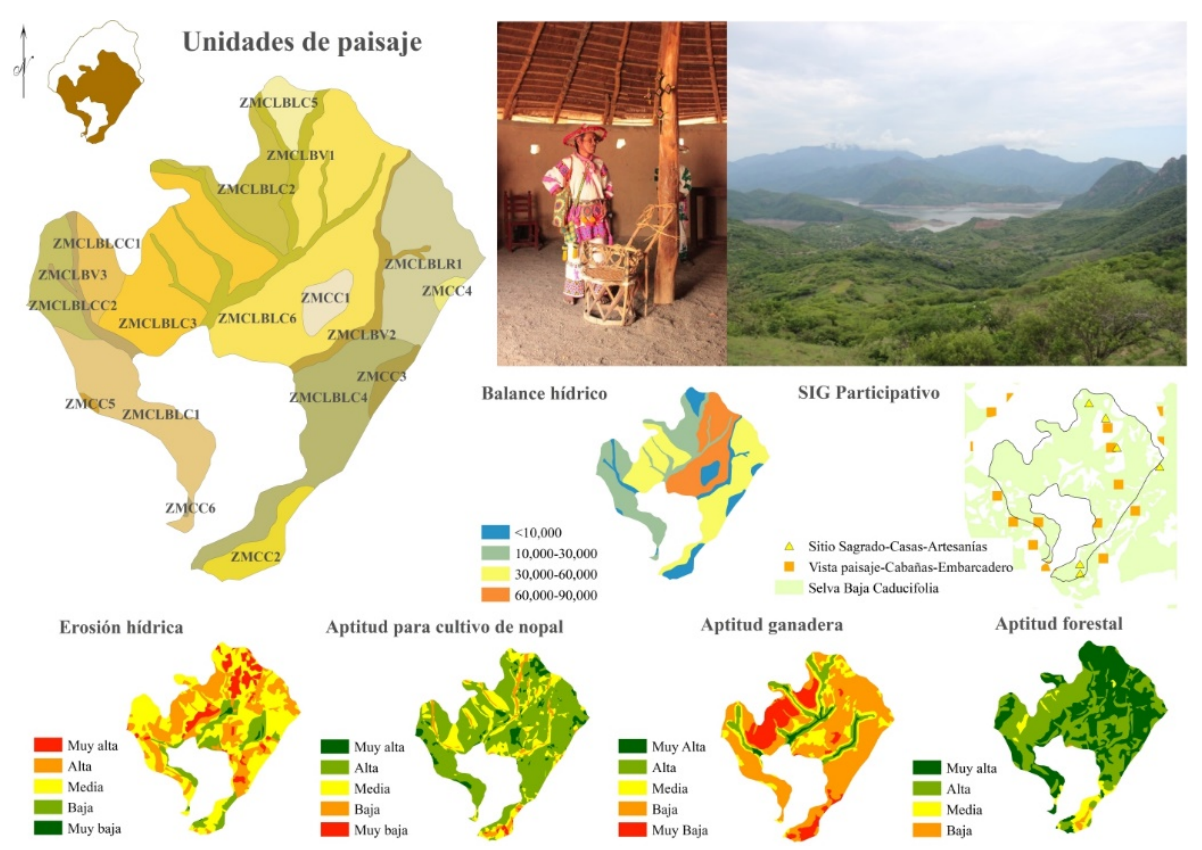

Figura 3. Diagnóstico integrado y participativo de la zona funcional media.

En lo que respecta a la aptitud, esta zona resultó con valores altos para el desarrollo forestal y la prestación de servicios ambientales en el 93\% y $89 \%$ de su área respectivamente. Por otro lado, el $80 \%$ de la zona no es apta para el establecimiento de asentamientos humanos, y es poco apta para el desarrollo de agricultura de temporal ( $65 \%$ con valores bajos), y ganadería extensiva ( $70 \%$ con valores bajos). No obstante, la actividad turística puede ser una buena opción al encontrarse con valores altos y medios en el $80 \%$ de su superficie.

La comunidad señaló que la zona tiene potencial natural, paisajístico y cultural, debido a su biodiversidad y panorámicas agradables, seguido de las oportunidades turísticas (aprovechamiento de recursos de la selva y del paisaje) y las actividades artesanales que ahí se desarrollan. En otro contexto, la comunidad detectó problemáticas similares a la zona anterior (sea la falta de agua, pérdida de cultivos, degradación, contaminación, erosión y remoción en masa), adicionándose la falta de dotación de servicios a los habitantes y turistas, el desempleo, la migración y la pérdida de cultura e identidad de los habitantes jóvenes (Tabla 5). 
Tabla 5

Diagnóstico participativo para la zona funcional media

\begin{tabular}{|c|c|c|c|c|c|c|c|c|c|c|c|c|}
\hline \multirow{3}{*}{$\begin{array}{c}\text { Zona } \\
\text { Funcional }\end{array}$} & \multirow{3}{*}{ Clave UP } & \multicolumn{11}{|c|}{ Diagnóstico participativo } \\
\hline & & \multicolumn{5}{|c|}{ Potencial } & \multicolumn{6}{|c|}{ Problemática } \\
\hline & & $N$ & $C E$ & $P$ & $T U$ & E & $A P C$ & $E R$ & $D C$ & $M D$ & $C I$ & $S$ \\
\hline \multirow{18}{*}{ Media } & ZMCC1 & $\mathrm{O}$ & & $\mathrm{O}$ & & & & $\mathrm{O}$ & & & & \\
\hline & ZMCC2 & $\mathrm{O}$ & $\mathrm{O}$ & $\mathrm{O}$ & $\mathrm{O}$ & $\mathrm{O}$ & $\mathrm{O}$ & $\mathrm{O}$ & $\mathrm{O}$ & $\mathrm{O}$ & $\mathrm{O}$ & $\mathrm{O}$ \\
\hline & ZMCC3 & & & & & & $\mathrm{O}$ & & $\mathrm{O}$ & & & \\
\hline & ZMCC4 & $\mathrm{O}$ & & $\mathrm{O}$ & & & & & & & & \\
\hline & ZMCC5 & & & & & & & & & & & \\
\hline & ZMCC6 & $\mathrm{O}$ & & $\mathrm{O}$ & & & $\mathrm{O}$ & & $\mathrm{O}$ & $\mathrm{O}$ & $\mathrm{O}$ & $\mathrm{O}$ \\
\hline & ZMCLBLC1 & $\mathrm{O}$ & & $\mathrm{O}$ & $\mathrm{O}$ & $\mathrm{O}$ & $\mathrm{O}$ & $\mathrm{O}$ & $\mathrm{O}$ & $\mathrm{O}$ & $\mathrm{O}$ & $\mathrm{O}$ \\
\hline & ZMCLBLC2 & $\mathrm{O}$ & & $\mathrm{O}$ & & & & & & & & \\
\hline & ZMCLBLC3 & $\mathrm{O}$ & & $\mathrm{O}$ & & & $\mathrm{O}$ & & $\mathrm{O}$ & & & \\
\hline & ZMCLBLC4 & $\mathrm{O}$ & $\mathrm{O}$ & $\mathrm{O}$ & $\mathrm{O}$ & $\mathrm{O}$ & $\mathrm{O}$ & & $\mathrm{O}$ & $\mathrm{O}$ & $\mathrm{O}$ & $\mathrm{O}$ \\
\hline & ZMCLBLC5 & $\mathrm{O}$ & $\mathrm{O}$ & $\mathrm{O}$ & $\mathrm{O}$ & $\mathrm{O}$ & $\mathrm{O}$ & & $\mathrm{O}$ & & & \\
\hline & ZMCLBLC6 & $\mathrm{O}$ & $\mathrm{O}$ & $\mathrm{O}$ & $\mathrm{O}$ & $\mathrm{O}$ & $\mathrm{O}$ & & $\mathrm{O}$ & $\mathrm{O}$ & $\mathrm{O}$ & $\mathrm{O}$ \\
\hline & ZMCLBLCC1 & $\mathrm{O}$ & & $\mathrm{O}$ & & & & & & & & \\
\hline & ZMCLBLCC2 & $\mathrm{O}$ & & $\mathrm{O}$ & & & $\mathrm{O}$ & & $\mathrm{O}$ & & & \\
\hline & ZMCLBLR1 & $\mathrm{O}$ & & $\mathrm{O}$ & & & $\mathrm{O}$ & & $\mathrm{O}$ & & & \\
\hline & ZMCLBV1 & $\mathrm{O}$ & & $\mathrm{O}$ & & & $\mathrm{O}$ & & $\mathrm{O}$ & & & \\
\hline & ZMCLBV2 & $\mathrm{O}$ & & $\mathrm{O}$ & $\mathrm{O}$ & $\mathrm{O}$ & $\mathrm{O}$ & $\mathrm{O}$ & $\mathrm{O}$ & & & \\
\hline & ZMCLBV3 & $\mathrm{O}$ & & $\mathrm{O}$ & & & $\mathrm{O}$ & & $\mathrm{O}$ & & & \\
\hline
\end{tabular}

$\mathrm{N}$ : natural, CE: cultural-educativo, P: paisajístico, TU: turístico, E: económico; APC: falta de agua y pérdida de cultivos, ER: erosión hídrica y remoción en masa, DC: degradación y contaminación, MD: migración y desempleo, CI: pérdida de cultura e identidad comunitaria, S: dotación de servicios; O: detectado.

Fuente: elaboración con base en taller y SIG participativo.

\section{Zona funcional baja}

Representa la zona con mayor modificación del ecosistema natural provocada por las actividades económicas de la población, generando degradación y fragmentación del hábitat nativo por medio de la deforestación y conversión de actividades productivas primarias (agropecuarias) a terciarias (asentamientos humanos). Esta zona recibe la menor cantidad de agua proveniente de la precipitación (10.43\%), a pesar de ello, se cuenta con el escurrimiento acumulado de la zona alta y media, por lo que recibe $657583.08 \mathrm{~m}^{3}$ de agua al año, ello genera un balance hídrico positivo (superávit) que es evidente en temporada de lluvias (junio-septiembre), (Figura 4).

La zona baja es también la que tiene menor pérdida de suelo, ya que el 55\% de su superficie reporta una tasa de pérdida del suelo entre 50 y 200 ton/ha/año, así como 
la de menor susceptibilidad ante peligros geológicos, debido a que en más del $70 \%$ de la zona se reportan valores bajos de remoción en masa y erosión. A diferencia de las zonas anteriores (alta y media), más del $50 \%$ de su superficie presenta alta susceptibilidad a peligros de origen hidrometeorológicos, especialmente a inundaciones.

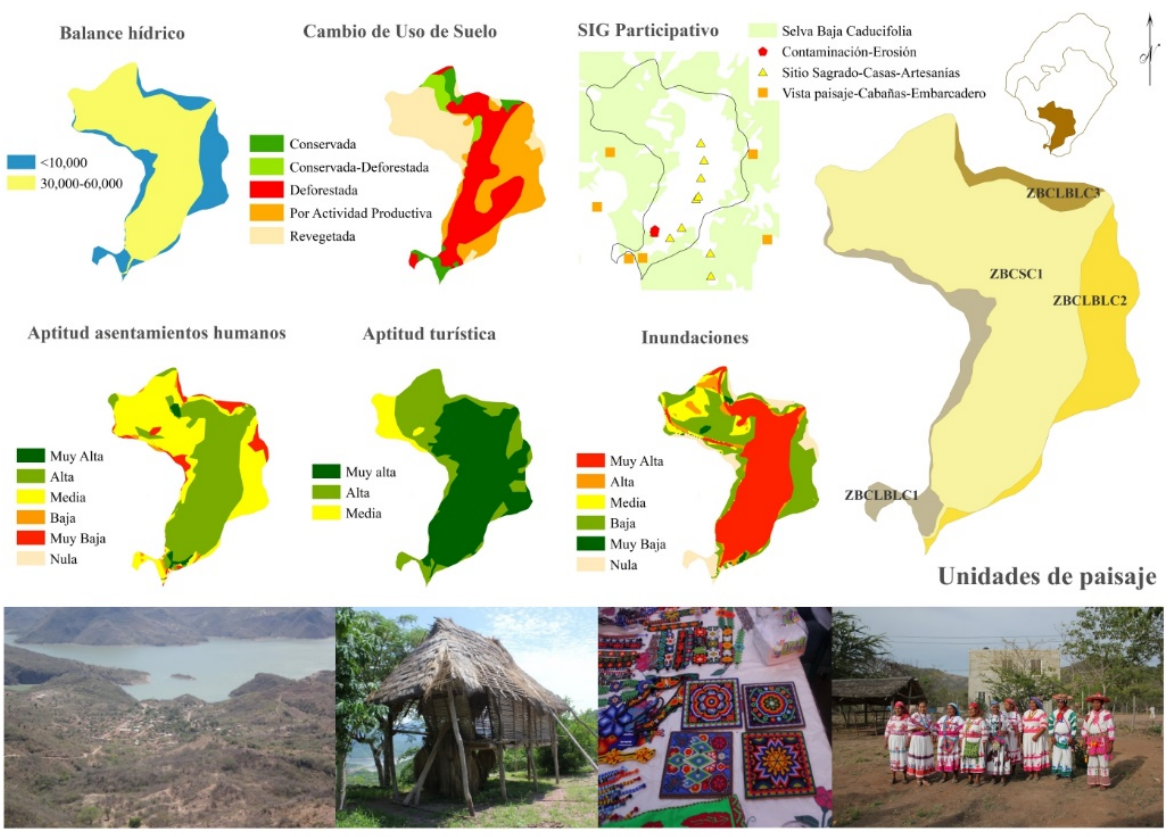

Figura 4. Diagnóstico integrado y participativo de la zona funcional baja.

Si bien los asentamientos humanos proliferan en esta zona, los resultados señalaron que solo el $42 \%$ de la superficie es apta para su establecimiento, mientras que el $60 \%$ presenta aptitud para el desarrollo de agricultura de temporal, cultivo de nopal y ganadería extensiva. Por lo tanto, nuevamente los valores más altos de aptitud están relacionados con actividades turísticas, ello equivale al $93 \%$ de su área (Tabla 6).

La opinión de los habitantes resaltó el potencial turístico, pues ya se tienen algunas ofertas de servicios como hospedaje, alimentación y recreación. Alberga también sitios de alta calidad cultural y artesanal, donde el turista puede adquirir nuevos conocimientos sobre el paisaje y la comunidad. Sin embargo, los grupos focales señalaron que prevalecen la mayoría de las problemáticas señaladas en las otras zonas funcionales (falta de agua y pérdida de cultivos). Aunque tiene disponibilidad de agua para uso de la comunidad, en la temporada de lluvias esta llega de forma torrencial, siendo deficiente su capacidad para captarla (Tabla 7). 
Tabla 6

Diagnóstico integrado para la zona funcional baja

\begin{tabular}{|c|c|c|c|c|c|c|c|c|c|c|c|c|c|}
\hline \multirow{3}{*}{$\begin{array}{c}\text { Clave de la } \\
\text { unidad de } \\
\text { paisaje }\end{array}$} & \multicolumn{13}{|c|}{ Diagnóstico integrado } \\
\hline & \multirow{2}{*}{ 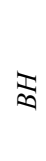 } & \multirow{2}{*}{$\tilde{v}$} & \multirow{2}{*}{ 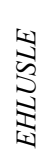 } & \multicolumn{3}{|c|}{ Susceptibilidad } & \multicolumn{7}{|c|}{ Aptitud } \\
\hline & & & & $\underset{\Sigma}{\geqq}$ & 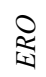 & $\gtreqless$ & $z_{1}$ & ॠ & ¿ & $\nabla$ & ல্ত & 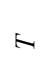 & $\underset{\mathbb{I}}{\underset{\sim}{7}}$ \\
\hline ZBCLBLC1 & $<$ & $\mathrm{D}$ & $\mathrm{A}$ & $\mathrm{M}$ & $\mathrm{M}$ & $\mathrm{N}$ & $A^{\prime}$ & $A^{\prime}$ & $\mathrm{A}$ & $\mathrm{B}$ & $\mathrm{B}$ & $\mathrm{A}$ & $\mathrm{M}$ \\
\hline ZBCLBLC2 & $<$ & $\mathrm{D}$ & A & M & $\mathrm{M}$ & $\mathrm{B}$ & M & A & A & B & $\mathrm{B}$ & $A^{\prime}$ & M \\
\hline ZBCLBLC3 & $<$ & $\mathrm{D}$ & $\mathrm{A}$ & M & $\mathrm{B}$ & $\mathrm{N}$ & $A^{\prime}$ & A & M & $\mathrm{B}^{\prime}$ & $\mathrm{B}$ & A & $B^{\prime}$ \\
\hline ZBCSC1 & $>$ & $\mathrm{D}$ & $\mathrm{A}$ & $\mathrm{B}$ & $\mathrm{B}^{\prime}$ & $A^{\prime}$ & $\mathrm{M}$ & $\mathrm{B}$ & A & M & $\mathrm{A}$ & $A^{\prime}$ & A \\
\hline
\end{tabular}

BH: balance hídrico, >: mayor a $10000 \mathrm{~m}^{3} /$ año, <: menor a $10000 \mathrm{~m}^{3} / \mathrm{año}$; CUS: cambio de uso del suelo, D: deforestada; EHLUSLE: erosión hídrica laminar USLE, RM: remoción en masa, ERO: erosión, INU: inundaciones, FN: forestal natural, PSA: prestación de servicios ambientales, $\mathrm{CN}$ : cultivo de nopal, AT: agrícola de temporal, GE: ganadera extensiva, T: turística, EAH: establecimiento de asentamientos humanos; A': muy alta, A:alta, M: media, B: baja, B': muy baja, $\mathrm{N}$ : nula.

Fuente: elaboración con base en los resultados del SIG y de campo.

Tabla 7

Diagnóstico participativo para la zona funcional baja

\begin{tabular}{|c|c|c|c|c|c|c|c|c|c|c|c|c|}
\hline \multirow{3}{*}{$\begin{array}{c}\text { Zona } \\
\text { Funcional }\end{array}$} & \multirow{3}{*}{$\begin{array}{c}\text { Clave } \\
U P\end{array}$} & \multicolumn{11}{|c|}{ Diagnóstico participativo } \\
\hline & & \multicolumn{5}{|c|}{ Potencial } & \multicolumn{6}{|c|}{ Problemática } \\
\hline & & $N$ & $C E$ & $P$ & $T U$ & $E$ & $A P C$ & $E R$ & $D C$ & $M D$ & $C I$ & $S$ \\
\hline \multirow{4}{*}{ Baja } & ZBCLBLC1 & $\mathrm{O}$ & & $\mathrm{O}$ & $\mathrm{O}$ & $\mathrm{O}$ & $\mathrm{O}$ & & $\mathrm{O}$ & $\mathrm{O}$ & $\mathrm{O}$ & $\mathrm{O}$ \\
\hline & ZBCLBLC2 & $\mathrm{O}$ & & $\mathrm{O}$ & & & $\mathrm{O}$ & & $\mathrm{O}$ & $\mathrm{O}$ & $\mathrm{O}$ & $\mathrm{O}$ \\
\hline & ZBCLBLC3 & $\mathrm{O}$ & & $\mathrm{O}$ & & & $\mathrm{O}$ & & $\mathrm{O}$ & $\mathrm{O}$ & $\mathrm{O}$ & $\mathrm{O}$ \\
\hline & ZBCSC1 & $\mathrm{O}$ & $\mathrm{O}$ & $\mathrm{O}$ & $\mathrm{O}$ & $\mathrm{O}$ & $\mathrm{O}$ & $\mathrm{O}$ & $\mathrm{O}$ & $\mathrm{O}$ & $\mathrm{O}$ & $\mathrm{O}$ \\
\hline
\end{tabular}

N: natural, CE: cultural-educativo, P: paisajístico, TU: turístico, E: económico; APC: falta de agua y pérdida de cultivos, ER: erosión hídrica y remoción en masa, DC: degradación y contaminación, MD: migración y desempleo, CI: pérdida de cultura e identidad comunitaria, S: dotación de servicios; O: detectado.

Fuente: elaboración con base en taller y SIG participativo.

Los participantes de los talleres indicaron la presencia de problemas como la degradación de suelo, la quema de basura, la erosión, la remoción en masa, el desempleo, la migración, la pérdida de cultura e identidad y la carencia en la dotación de servicios. 


\section{Optimización del uso del territorio por zona funcional}

A partir del conocimiento del estado socioambiental de las unidades de paisaje físicogeográfico, se identificaron los usos de suelo principales, secundarios y terciarios, definiendo su compatibilidad, proponiendo nueve diferentes usos para optimizar el uso del suelo dentro de las zonas funcionales de la microcuenca (Tabla 8).

Tabla 8

Propuesta de optimización del uso por unidad de paisaje

\begin{tabular}{|c|c|c|c|c|c|c|}
\hline \multirow{3}{*}{$\begin{array}{c}\text { Zona } \\
\text { funcional }\end{array}$} & \multirow{3}{*}{$\begin{array}{c}\text { Clave } \\
\text { UP }\end{array}$} & \multicolumn{5}{|c|}{ Propuesta } \\
\hline & & \multicolumn{3}{|c|}{ Uso del suelo actual } & \multirow{2}{*}{ COMP } & \multirow{2}{*}{$\begin{array}{c}\text { Usos } \\
\text { propuestos }\end{array}$} \\
\hline & & Principal & Secundario & Terciario & & \\
\hline \multirow{17}{*}{ Alta } & $\mathrm{ZACC1}$ & SBC & BNLE & SBCP & A & $\mathrm{CO}-\mathrm{T}, \mathrm{CO}$ \\
\hline & ZACC2 & SBC & PI & SBCP & M & $\mathrm{CO}-\mathrm{T}, \mathrm{CO}$ \\
\hline & ZACC3 & PI & SBCP & SBC & $\mathrm{B}$ & $\mathrm{CO}-\mathrm{T}, \mathrm{CO}$ \\
\hline & ZACC4 & SBCP & & & A & $\mathrm{CO}-\mathrm{T}$ \\
\hline & ZACC5 & $\mathrm{SBCP}$ & $\mathrm{SBC}$ & & A & $\mathrm{CO}-\mathrm{T}, \mathrm{CO}$ \\
\hline & ZACC6 & SBC & SBCP & & A & $\mathrm{CO}$ \\
\hline & ZACC7 & $\mathrm{SBCP}$ & SBC & & A & $\mathrm{CO}$ \\
\hline & ZACC8 & SBCP & & & A & $\mathrm{CO}$ \\
\hline & ZACLBLC1 & SBC & PI & SBCP & M & $\mathrm{CO}-\mathrm{T}, \mathrm{CO}$ \\
\hline & ZACLBLCC1 & SBC & SBCP & $\mathrm{A}$ & M & $\mathrm{CO}-\mathrm{T}, \mathrm{CO}$ \\
\hline & ZACLBLR1 & SBCP & PI & SBC & M & $\mathrm{CO}-\mathrm{T}, \mathrm{CO}$ \\
\hline & ZACLBV1 & SBC & & & $\mathrm{A}$ & $\mathrm{CO}$ \\
\hline & ZACLBV2 & SBC & PI & A & M & $\mathrm{CO}, \mathrm{AP}$ \\
\hline & ZACLBV3 & $\mathrm{SBC}$ & $\mathrm{SBCP}$ & & $\mathrm{A}$ & $\mathrm{CO}$ \\
\hline & ZACLBV4 & SBC & PI & & M & $\mathrm{CO}-\mathrm{T}, \mathrm{CO}$ \\
\hline & ZACLBV5 & $\mathrm{SBC}$ & $\mathrm{SBCP}$ & & A & $\mathrm{CO}$ \\
\hline & ZACSC1 & PI & $\mathrm{SBC}$ & & $\mathrm{A}$ & AP-T, AP \\
\hline \multirow{11}{*}{ Media } & $\mathrm{ZMCC1}$ & SBCP & PI & SBC & $\mathrm{M}$ & $\mathrm{CO}$ \\
\hline & $\mathrm{ZMCC} 2$ & $\mathrm{AH}$ & $\mathrm{SBCP}$ & SBC & B & $\begin{array}{l}\mathrm{CO}, \mathrm{AP}, \mathrm{AP}-\mathrm{T}, \\
\mathrm{AH}-\mathrm{T}\end{array}$ \\
\hline & ZMCC3 & SBCP & A & PI & M & $\mathrm{CO}$ \\
\hline & ZMCC4 & SBCP & & & A & $\mathrm{CO}$ \\
\hline & ZMCC5 & SBCP & & & A & $\mathrm{CO}$ \\
\hline & ZMCC6 & SBC & $\mathrm{AH}$ & & M & $\mathrm{CO}, \mathrm{AH}-\mathrm{T}$ \\
\hline & ZMCLBLC1 & SBCP & A & SBC & M & $\begin{array}{l}\mathrm{CO}, \mathrm{A}^{*}, \mathrm{~A}^{*}-\mathrm{T} \\
\mathrm{AH}-\mathrm{T}, \mathrm{AP}-\mathrm{T}\end{array}$ \\
\hline & ZMCLBLC2 & SBC & SBCP & & A & $\mathrm{CO}$ \\
\hline & ZMCLBLC3 & $\mathrm{SBC}$ & A & SBCP & M & $\mathrm{CO}, \mathrm{A}^{*}$ \\
\hline & ZMCLBLC4 & SBCP & $\mathrm{AH}$ & A & M & $\begin{array}{l}\mathrm{CO}, \mathrm{CO}-\mathrm{T}, \mathrm{AP}, \\
\mathrm{A}^{*}, \mathrm{P}, \mathrm{AH}-\mathrm{T}\end{array}$ \\
\hline & ZMCLBLC5 & SBC & A & & M & $\mathrm{CO}, \mathrm{CO}-\mathrm{T}$ \\
\hline
\end{tabular}


Continuación Tabla 8

\begin{tabular}{|c|c|c|c|c|c|c|}
\hline \multirow{3}{*}{$\begin{array}{c}\text { Zona } \\
\text { funcional }\end{array}$} & \multirow{3}{*}{$\begin{array}{c}\text { Clave } \\
U P\end{array}$} & \multicolumn{5}{|c|}{ Propuesta } \\
\hline & & \multicolumn{3}{|c|}{ Uso del suelo actual } & \multirow{2}{*}{ COMP } & \multirow{2}{*}{$\begin{array}{c}\text { Usos } \\
\text { propuestos }\end{array}$} \\
\hline & & Principal & Secundario & Terciario & & \\
\hline \multirow{7}{*}{ Media } & ZMCLBLC6 & SBC & A & SBCP & M & $\begin{array}{l}\text { AH-T, } A^{*}, A^{*}- \\
\text { T, CO, CO-T, } \\
\text { AP-T, P }\end{array}$ \\
\hline & ZMCLBLCC1 & SBC & & & A & $\mathrm{CO}$ \\
\hline & ZMCLBLCC2 & SBC & $\mathrm{SBCP}$ & A & M & $\mathrm{CO}$ \\
\hline & ZMCLBLR1 & SBC & $\mathrm{SBCP}$ & A & M & $\begin{array}{l}\mathrm{CO}, \mathrm{CO}-\mathrm{T}, \mathrm{A}_{-} \\
\mathrm{T}, \mathrm{P}\end{array}$ \\
\hline & ZMCLBV1 & $\mathrm{SBC}$ & A & $\mathrm{SBCP}$ & M & $\begin{array}{l}\mathrm{CO}, \mathrm{CO}-\mathrm{T}, \mathrm{A}^{*}, \\
\mathrm{P}\end{array}$ \\
\hline & ZMCLBV2 & $\mathrm{SBC}$ & $\mathrm{SBCP}$ & AT & M & $\begin{array}{l}\mathrm{CO}, \mathrm{CO}-\mathrm{T}, \mathrm{A}^{*}, \\
\text { P-T }\end{array}$ \\
\hline & ZMCLBV3 & SBC & SBCP & AT & M & $\mathrm{CO}, \mathrm{A}^{*}$ \\
\hline \multirow{4}{*}{ Baja } & ZBCLBLC1 & $\mathrm{AH}$ & SBCP & SBC & B & $\begin{array}{l}\text { CO-T, CO, } \\
\text { AH-T, A* }\end{array}$ \\
\hline & ZBCLBLC2 & $\mathrm{AH}$ & SBCP & A & B & $\begin{array}{l}\mathrm{CO}, \mathrm{AH}-\mathrm{T}, \mathrm{A}^{*}, \\
\mathrm{~A}^{*} \mathrm{-T}\end{array}$ \\
\hline & ZBCLBLC3 & $\mathrm{SBC}$ & $\mathrm{AH}$ & A & M & $\begin{array}{l}\text { CO, P, A*, } \\
\text { AH-T }\end{array}$ \\
\hline & ZBCSC1 & $\mathrm{AH}$ & SBCP & SBC & M & $\begin{array}{l}\mathrm{CO}, \mathrm{AH}-\mathrm{T}, \mathrm{A}^{*}, \\
\mathrm{P}, \mathrm{AP}\end{array}$ \\
\hline
\end{tabular}

BNLE: bosque de latifoliadas-encino, SBC: selva baja caducifolia, SBCP: selva baja caducifolia perturbada, PI: pastizal inducido, A: agricultura de temporal, AH: asentamientos humanos; COMP: compatibilidad, A: alta, M: media, B: baja; CO: conservación, CO-T: conservación-turístico, AP: agropecuario, AP-T: agropecuario-turístico, A*: agrícola, A*-T: agrícola-turístico. P: pecuario, P-T: pecuario-turístico, AH-T: asentamiento-turístico.

Fuente: elaboración propia.

La cartografía resultante muestra que las propuestas de uso del suelo para la zona alta coinciden con su potencial natural, fomentando la conservación del ecosistema nativo de la microcuenca, ya que en las unidades de paisaje predominan las propuestas de uso para conservación ( $\mathrm{CO}$ ) y conservación-turístico (CO-T). Las propuestas consideran acciones como la restricción y manejo del pastoreo de ganado, la revegetación con especies nativas, el fomento al crecimiento vegetal, la siembra de barreras vivas, la construcción de jagüeyes, canales de llamada, tinas ciegas, media lunas y presas filtrantes de costales con material de la región, la aplicación de técnicas de surcado al contorno y de terráceo, el manejo de escurrimientos, el aprovechamiento de productos forestales no maderables, el acondicionamiento de senderos y mamparas interpretativas, así como, miradores y sitios de avistamiento (Figura 5). 


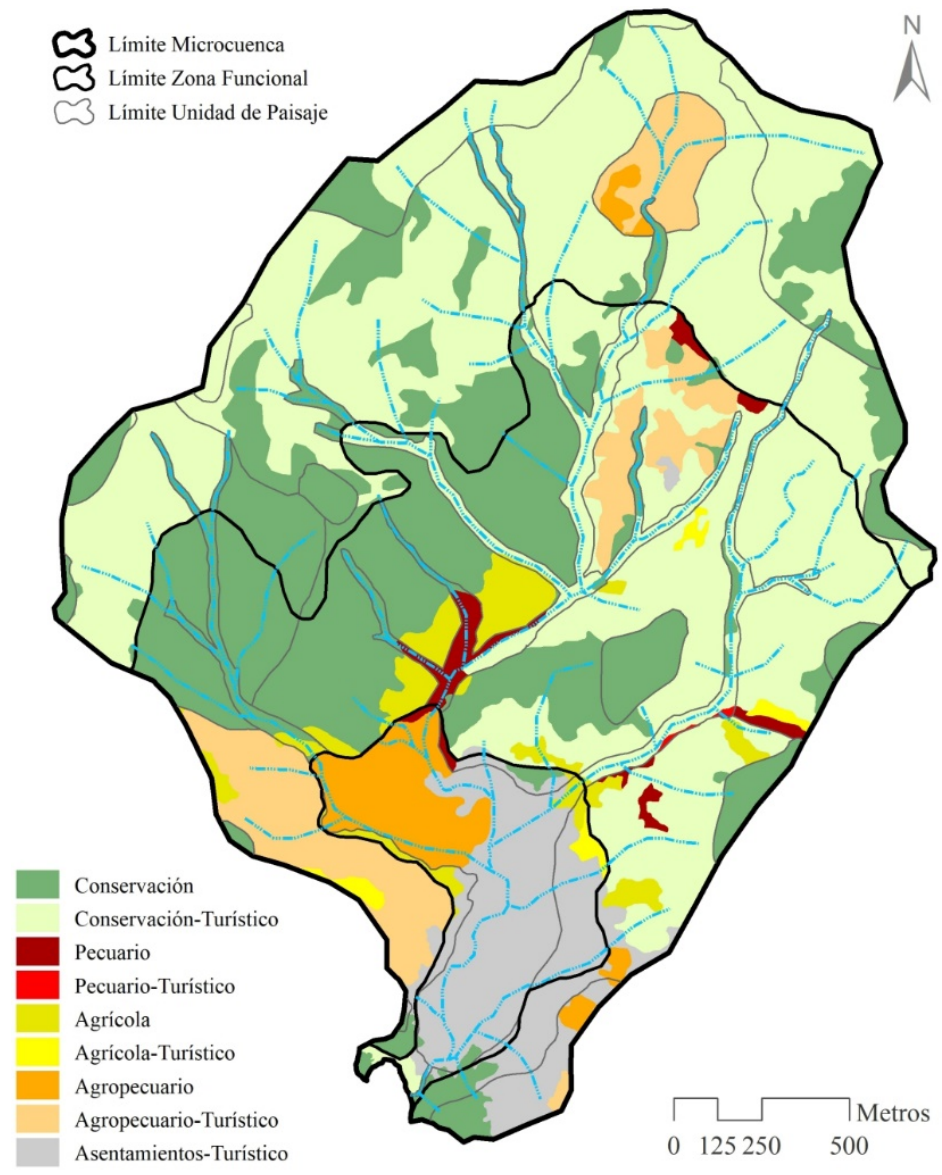

Figura 5. Propuesta de optimización del uso del suelo.

La zona media, al ser una zona con posibilidades de desarrollo de actividades de conservación del paisaje natural y el desarrollo de actividades socio-productivas, las propuestas de uso en las unidades de paisaje que la conforman estarían dirigidas a la conservación (CO), conservación-turístico (CO-T) y agropecuario-turístico (AP-T). Esas unidades contemplan el desarrollo de acciones restrictivas y de manejo del pastoreo, la revegetación con especies nativas, el fomento al crecimiento vegetal, la siembra de barreras vivas, la construcción de jagüeyes, canales de llamada, tinas ciegas, media lunas y presas filtrantes de costales con material de la región.

Las acciones también consideran el manejo de escurrimientos, surcado al contorno y terráceo, el aprovechamiento de productos forestales no maderables, el acondicionamiento de senderos y mamparas interpretativas, la construcción de miradores 
y sitios de avistamiento, el uso y manejo de las compostas, abonos verdes y estiércoles (abonos orgánicos), la conservación del sistema milpa y la siembra de policultivos adaptados a la microcuenca, retomando las técnicas de labranza tradicional conservadas ancestralmente por la comunidad.

Finalmente, los usos de suelo propuestos para la zona baja coinciden con su potencial para el desarrollo de actividades socio-productivas, donde predominan las propuestas de uso para el establecimiento de asentamientos humano-turísticos (AHT) y agropecuarios (AP). Esas propuestas promueven el desarrollo de actividades de manejo del pastoreo, la revegetación con especies nativas, la construcción de tinas ciegas, medias lunas y jagüeyes, la aplicación de abonos orgánicos como el estiércol, el aprovechamiento de productos forestales no maderables para la diversificación de alimentos y productos para la comunidad.

Las propuestas también consideran acciones encaminadas a conservar la construcción tradicional de viviendas y su imagen rural, la implementación de granjas ecológicas y huertos familiares, el desarrollo de representaciones culturales en la comunidad, el acondicionamiento de mamparas, senderos y miradores interpretativos, la señalización adecuada de los sitios culturales y artesanales, la valoración económica y difusión de los servicios comunitarios, y el manejo de residuos sólidos generados por los visitantes y habitantes de la microcuenca.

\section{Conclusiones}

La investigación representó una metodología viable y repetible para abordar la planeación territorial a escala de gran detalle en una microcuenca, permitió generar estrategias de gestión, planeación y manejo a partir de la identificación de su estado, dinámica, procesos, potenciales y problemáticas socioambientales, teniendo como principal herramienta las plataformas de SIG.

Las propuestas de optimización del uso del territorio derivadas del análisis, coincidieron con el potencial y las demandas para corregir o disminuir las problemáticas específicas de cada unidad de paisaje físico-geográfico y zona funcional, prevaleciendo en la zona alta propuestas dirigidas a la conservación y turismo, en la zona media al desarrollo de actividades agropecuarias, de conservación y turismo, y en la zona baja propuestas para el desarrollo agropecuario, turístico y de asentamientos humanos.

El trabajo realizado muestra la pertinencia de delimitar las zonas funcionales para estudiar una microcuenca, ya que mediante su correcta diferenciación utilizando plataformas de SIG, análisis geomorfológico y trabajo de campo, es posible evaluar de manera integral su dinámica, evolución y funcionalidad, eso derivó en la óptima planeación de actividades para su manejo. 
Asimismo, fue posible contemplar a las cuencas hidrográficas como unidades territoriales cuyo funcionamiento y estructura es factible evaluar por medio de la delimitación de unidades espaciales de menor superficie o unidades de paisaje físicogeográfico, aumentando el detalle en el estudio de sus condiciones socio-ambientales, y así realizar un análisis puntual de las problemáticas y potenciales que presenta.

Es importante continuar con esquemas de gestión y divulgación que funjan como guía y herramienta para direccionar la toma de decisiones. Fomentar el monitoreo y la evaluación de las acciones para impulsar la adecuada planeación de su territorio, sea de manera interna por parte de la comunidad o de manera externa a través de los organismos gubernamentales (federal, estatal y municipal), e instituciones académicas y educativas que han intervenido en la microcuenca.

Finalmente, se sugiere impulsar y mejorar los programas enfocados a la producción de maíz, animales, dotación de servicios de luz y energía eléctrica, abasto de alimentos básicos, artesanías, salud, higiene, desarrollo social, ambiental, educativo, de reforestación, de empleo temporal y de investigaciones académicas, todo ello con base en el conocimiento propio de la microcuenca y el cumplimiento de las acciones y metas planteadas para la optimización del uso de su territorio.

\section{Bibliografía}

Bertani, L.A. (2011). "Evaluación geoecológica de los paisajes del Departamento Minas (Provincia de Neuquén), para el estudio de la degradación de la tierra" [en línea], tesis doctoral, Universidad Nacional de La Plata, Facultad de Humanidades y Ciencias de la Educación, La Plata, Argentina.

https://doi.org/10.35537/10915/3209.

Bocco, G.; Mendoza, M.; Plascencia, H.; Alcántara, P. y Rosete, F., (2010). Análisis de la Aptitud Territorial. Una perspectiva biofísica, SEMARNAT-INE, México. https://doi.org/10.22201/ciga.9789688179178e.2011.

Bocco, G.; Mendoza, M.; Priego, A. y Burgos, A. (2009). La cartografía de los sistemas naturales como base geográfica para la planeación territorial. Una revisión de la bibliografia, SEMARNAT-INE-UNAM-CIGA, Primera Edición, México. https://doi.org/10.22201/ciga.9789688179208p.2009.

Colín, G.G.; Ibáñez, C.L.A.; Reyes, S.J. y Arteaga, R.R. (2013). Diagnóstico de la erosión hídrica de la cuenca del Río Pichucalco, Universidad Autónoma de Chapingo, Texcoco, Estado de México. Disponible en:

http://dx.doi.org/10.5154/r.inagbi.2013.04.002.

Cotler, H. (2007). El Manejo Integral de Cuencas en México. Estudios y reflexiones para orientar la política ambiental, segunda edición, SEMARNAT-INE, México. Recuperado de https://agua.org.mx/. 
DOF (Diario Oficial de la Federación). (2015). Norma Oficial Mexicana NOM-011CONAGUA-2015. Conservación del recurso agua-Que establece las especificaciones y el método para determinar la disponibilidad media anual de las aguas nacionales. Publicada el 27 de marzo de 2015. Recuperado de www.dof.gob.mx.

Fernández, M.E.; Ávila, A.P. y Taylor, H.L. (2009). SIG-P y experiencias de cartografía social en la ciudad de Bogotá (Colombia), Grupo SIG Participativo $(S I G P)$, Universidad Nacional de Colombia. Recuperado de www.observatoriogeograficoamericalatina.org.mx.

Franch, P.I.; Priego, S.A.G.; Bollo, M.M.; Cancer, P.L. y Bautista, Z.F. (2015). “Aplicación de los paisajes fisicogeográficos en un sector de la cordillera ibérica: la cuenca del Río Martín, Aragón, España”, Interciencia, 40(6): 381-389. Recuperado de https://www.redalyc.org/articulo.oa? id=339/33938675004.

García, C.W. (2006). El Sistema Complejo de la Cuenca Hidrográfica, Facultad de Ciencias Humanas, Universidad de Colombia, Medellín, Colombia. Recuperado de www.medellin.unal.edu.co.

Geilfus, F. (2009). 80 herramientas para el desarrollo participativo: diagnóstico, planificación, monitoreo, evaluación, Instituto Interamericano de Cooperación para la Agricultura, Octava reimpresión, San José, Costa Rica. Recuperado de http://ejoventut.gencat.cat/permalink/aac2bb0c-2a0c-11e4-bcfe-005056924a59.

Hasdenteufel, P., Rodríguez, M.J.M., Baume, O. y Torres Gomez de Cadiz, R.J. (2008). "La Geoecología como herramienta para la gestión ambiental-Estudio de caso de la cuenca hidrográfica superficial del Río Quibú. Provincia Ciudad de la Habana. Cuba", Revista Universitaria de Geografía, 17: 309-329. Recuperado de https://www.redalyc.org/articulo.oa?id=3832/383239098012.

Hernández, S.J.R.; Bollo, M.M.; Méndez, L.A.P. y Figueroa, M.E.J.M. (2012). El relieve, los paisajes físicos-geográficos y la aptitud natural de las unidades de gestión en el ordenamiento ecológico territorial, Instituto de Geografía, Centro de Investigaciones en Geografía Ambiental, UNAM, México. Recuperado de https://www.redalyc.org/articulo.oa?id=539/53907604.

Jaque, C.E. (2010). Diagnóstico de los paisajes mediterráneos costeros. Cuenca del Rio Andalién, Chile, Facultad de Arquitectura, Urbanismo y Geografía, Universidad de Concepción, Chile. Recuperado de https://dialnet.unirioja.es/servlet/articulo? codigo $=3605452$.

López, R.A.; Lozano, R.P. y Sierra, C.P.C. (2012). Criterios de zonificación ambiental usando técnicas participativas y de información: estudio de caso zona costera del departamento del atlántico, Instituto de Investigaciones Marinas y Costeras INVERMAR. Punta de Betín, Santa Marta, Colombia. Recuperado de http://hdl.handle.net/1834/4432. 
Martínez, M.M. (2005). Estimación de la erosión del suelo, SAGARPA-COLPOSINCA RURAL, México. Recuperado de https://docplayer.es/24902826-Estimacion-de-la-erosion-del-suelo-dr-mario-martinez-menez.html.

Muñoz, M.D. y Azócar, G. (2014). "Incorporación del paisaje en la gestión del territorio de la Patagonia Chilena”, Urbano, 17(30): 34-59, Universidad del Bío Bío, Concepción, Chile. Recuperado de

https://www.redalyc.org/articulo.oa?id=198/19836174004.

Nogué, J. y Sala, P. (2008). "El paisaje en la ordenación del territorio. Los catálogos de paisaje de Cataluña", Cuadernos geográficos, (43): 69-98, Universidad de Granada, Granada, España. Recuperado de

https://revistaseug.ugr.es/index.php/cuadgeo/article/view/1110.

Noriega, F.S. (2008). El Territorio como Concepto Operativo. La Ordenación del Territorio, España. Recuperado de https://es.scribd.com/document/381192911/1-Noriega-Fernandez-El-Territorio-Como-Concepto-Operativo-Noriega-Fernandez-1.

Palacio, P.J.L.; Sánchez, S.M.T.; Casado, I.J.M.; Propin, F.E.; Delgado, C.J.; Velázquez, M.A.; Camacho, R.C.G. (2004). Indicadores para la caracterización y ordenamiento del territorio, UNAM, INE, CONANP, CONABIO, SEGOB, INEGI, SEDESOL, primera edición, México. Recuperado de https://www.researchgate.net/publication/288840782_Indicadores_para_la_Caracterizacion_y_Ordenamiento del Territorio.

Reyes, E.A. y Campos, V.M. (2014). Bases para la estandarización en la elaboración de atlas de riesgos y Catálogo de datos geográficos para representar el riesgo, SEDATU. Recuperado de https://www.gob.mx/cms/uploads/attachment/file/40838/Bases_AR_PRAH_2014.pdf.

Salinas, E.M.E. (2008). El ordenamiento territorial: experiencias internacionales, SEMARNAT-INE-UDG, México. Disponible en:

http://bibliotecasibe.ecosur.mx/sibe/book/000050374.

Vargas, U.G. (2012). "Espacio y territorio en el análisis geográfico", Reflexiones, 91: 313-326, Universidad de Costa Rica, San José, Costa Rica. Disponible en: http://www.redalyc.org/articulo.oa?id=72923937025.

Valdés, C.A.C. y Hernández, G.J.A. (2018). "Zonas funcionales y unidades de paisaje físico-geográfico en la microcuenca Potrero de la Palmita, Nayarit, México”, Revista Geográfica de América Central, (60): 189-229, enero-junio. https://doi.org/10.15359/rgac.60-1.7. 